\title{
Giant murals of Baja California: new regional archaeological perspectives
}

\section{A. Watchman, M. DE la L. GuTiÉRRez \& M. Hernandez Llosas*}

FIGURE 1 (right). Ladders are necessary to enable researchers to examine the nature of the paints and to collect samples. Dr Valerie Magar atop a structure of ladders at the San Borjitas site.
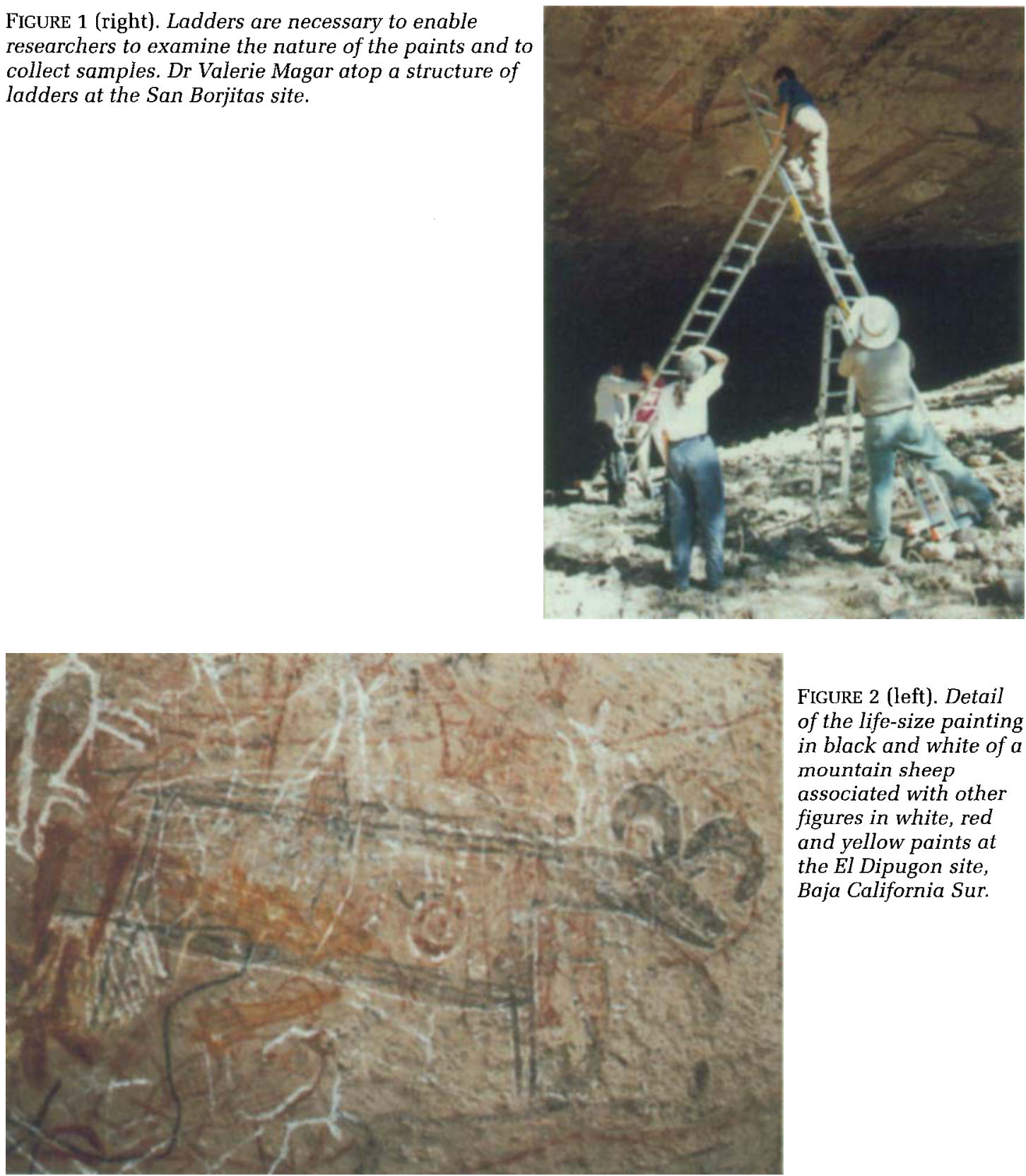

FIGURE 2 (left). Detail of the life-size painting in black and white of a mountain sheep associated with other figures in white, red and yellow paints at the El Dipugon site, Baja California Sur.

* Watchman, Department of Archaeology \& Natural History, Research School of Pacific \& Asian Studies, Australian National University, Canberra ACT 0200, Australia. Gutierrez, Instituto Nacional de Antropologia e Historia, Centro INAH Baja California Sur, La Paz, Mexico. Llosas, Department of Archaeology, Institute of Anthropological Sciences, University of Buenos Aires, Buenos Aires, Argentina. 
A new regional archaeological project in the relatively unexplored Sierra de Guadalupe in Baja California Sur, Mexico, commenced in 2001 (Gutiérrez 2000). This project, concerning the regional archaeological perspectives of the giant mural paintings of Baja California Sur, is funded principally by the Consejo Nacional de Ciencia y Tecnología and the Instituto Nacional de Antropología e Historia (CONACyT/INAH). The National Geographic Society is funding the radiocarbon dating of the paintings (Grant \#7130-01).

This international collaborative project between archaeologists and geoscientists aims to obtain information from the giant mural paintings and from excavations in the same rock shelters the better to understand the social interactions of prehistoric people in the rugged desert environment. The major objectives are to locate sites of rock paintings and prehistoric human occupation, to explore the characteristics of these sites in terms of their environmental settings, to determine the functions, spatial distribution, use of resources and other cultural activities among rock art sites, to define the analytical units for rock art in order to refine the general definitions of the giant mural sub-styles, and to relate the rock art and archaeological sites of the Sierra de Guadalupe to similar locations in the surrounding sierras.

Rock shelters in Baja California are well known for the fabulously spectacular paintings of giant humans and animals (FIGURES 1,2), mostly in red and black, and also in white and yellow (Crosby
1984). The giant human paintings recognized as belonging to the San Francisco and Guadalupe styles (Crosby 1984) probably have temporal and geographic distributions, and may have had particular cultural affinities, but have never been dated systematically. In collaboration with the Rafter Radiocarbon Laboratory, New Zealand, we are identifying the organic paint binders and obtaining age determinations of that source of carbon. Our preliminary dating results indicate that the giant mural painting tradition started approximately 5500 years ago (TABLE 1 ). This is consistent with other results for large humans painted in shelters in the Sierra de San Francisco (Fullola et al. 1991; 1994; Gutiérrez \& Hyland in press). Our observations also indicate a continuous history of rock painting at many sites with a sequence of different motifs possibly including repainting which has extended over many years. The reasons for the variability between sites as well as the consistency of use of certain motif forms across a wide geographic area is an intriguing aspect on which our systematic approach will shed light.

Acknowledgements. We thank the Consejo Nacional de Ciencia y Tecnología, Instituto Nacional de Antropología e Historia (CONACyT/INAH), and the National Geographic Society (Grant \#7130-01) for funds which cover the field work and radiocarbon assays. Dawn Chambers, Christine Prior and Rodger Sparks at the Rafter Radiocarbon Laboratory are collaborating with stable isotopic and radiocarbon analyses. Watchman is supported by an Australian Research Council Fellowship.

$\begin{array}{llcc}\begin{array}{l}\text { site and sample } \\ \text { identity }\end{array} & \text { motif description } & \begin{array}{c}\text { stable carbon isotope } \\ \text { composition (\%) }\end{array} & \begin{array}{c}\text { radiocarbon age } \\ \text { (years BP) }\end{array} \\ \text { San Borjitas 11 } & \text { black human (SBS) } & \mathbf{- 1 6 \cdot 0} & \mathbf{5 5 2 5 \pm 7 5} \\ \text { San Borjitas 3 } & \text { black human (SFS) } & \mathbf{- 1 9 \cdot 3} & \mathbf{5 3 2 5 \pm 9 5} \\ \text { La Trinidad 5 } & \text { red deer } & \mathbf{- 1 2 \cdot 6} & \mathbf{5 2 2 5 \pm 8 5} \\ \text { San Borjitas 5 } & \text { red human (SBS) } & \mathbf{- 1 3 \cdot 0} & \mathbf{5 0 2 5 \pm 7 5} \\ \text { El Pilo 3 } & \text { white deer } & \mathbf{- 1 4 . 5} & \mathbf{4 7 9 0 \pm 7 0}\end{array}$

TABLE 1. Preliminary age determinations and stable carbon isotope compositions for organic binders in giant mural paint samples, Sierra de Guadalupe, Mexico (SBS = San Borjitas Style, SFS = San Francisco Style). 